\title{
Scintillation Arcs Shed Light on Scattering from Planar Plasma Sheets
}

\author{
Daniel R. Stinebring \\ Oberlin College, Physics and Astronomy Department \\ 110 North Professor St, Oberlin, OH, 44074, USA \\ email: dan.stinebring@oberlin.edu
}

\begin{abstract}
Scintillation arcs provide an unprecedented degree of detail into the scattering of radio waves from pulsars. We review evidence that has emerged over the last fifteen years that: a) the scattering of many nearby pulsars is dominated by one or several relatively thin "screens" of material, b) the resulting image on the sky is highly linear, with axial ratios at least as high as 10:1, and c) this arrangement is persistent for at least one source (B1133+16) for at least 25 years. We expand on the idea of Pen and Levin (2014) and previous authors that such scattering may be caused by linear sheets of plasma seen nearly edge-on. Further analysis of such scintillation arcs, including new work on multi-frequency, multi-epoch observations, should help elucidate the astrophysical nature of these ubiquitous scattering entities, which are currently not convincingly linked with any known structures.
\end{abstract}

Keywords. pulsars: general, pulsars: individual (B1133+16), ISM: general, ISM: bubbles, ISM: structure

\section{Introduction}

In the paper in which we reported the discovery of scintillation arcs (Stinebring et al. 2001), we noted that several nearby pulsars exhibited scintillation arcs with sharp outer boundaries. Further work revealed this to be a more general feature (Fig. 1). As theoretical understanding progressed (Walker et al. 2004; Cordes et al. 2006) this became more puzzling because a quasi-circular scattering disk on the sky will produce a scintillation arc with a filled interior.

Furthermore, as we have shown for the relatively nearby pulsar B1133+16 (Fig. 2) the scintillation arcs are distinct from each other and constant in curvature for at least 25 years.

An important additional piece of evidence for nearly linear scattering structures on the sky was provided by Brisken et al. 2010.

In this paper, which marked the birth of scintillometry (scintillation arcs + very long baseline interferometry), they were able to use an analysis of the cross-spectrum between Arecibo and the Green Bank Telescope to produce a highly detailed model of the scattering structures (Fig. 3).

\section{Planar sheet model of Pen and Levin (2014)}

Pen and Levin (2014), building on work that stretched back more than 25 years (e. g. Romani, Blandford, and Cordes 1987) proposed that the linear features commonly seen in scintillation arc analyses were due to approximately planar sheets seen nearly edge-on. Furthermore, they proposed that these sheets might be caused by magnetic reconnection in ancient supernova shells. 

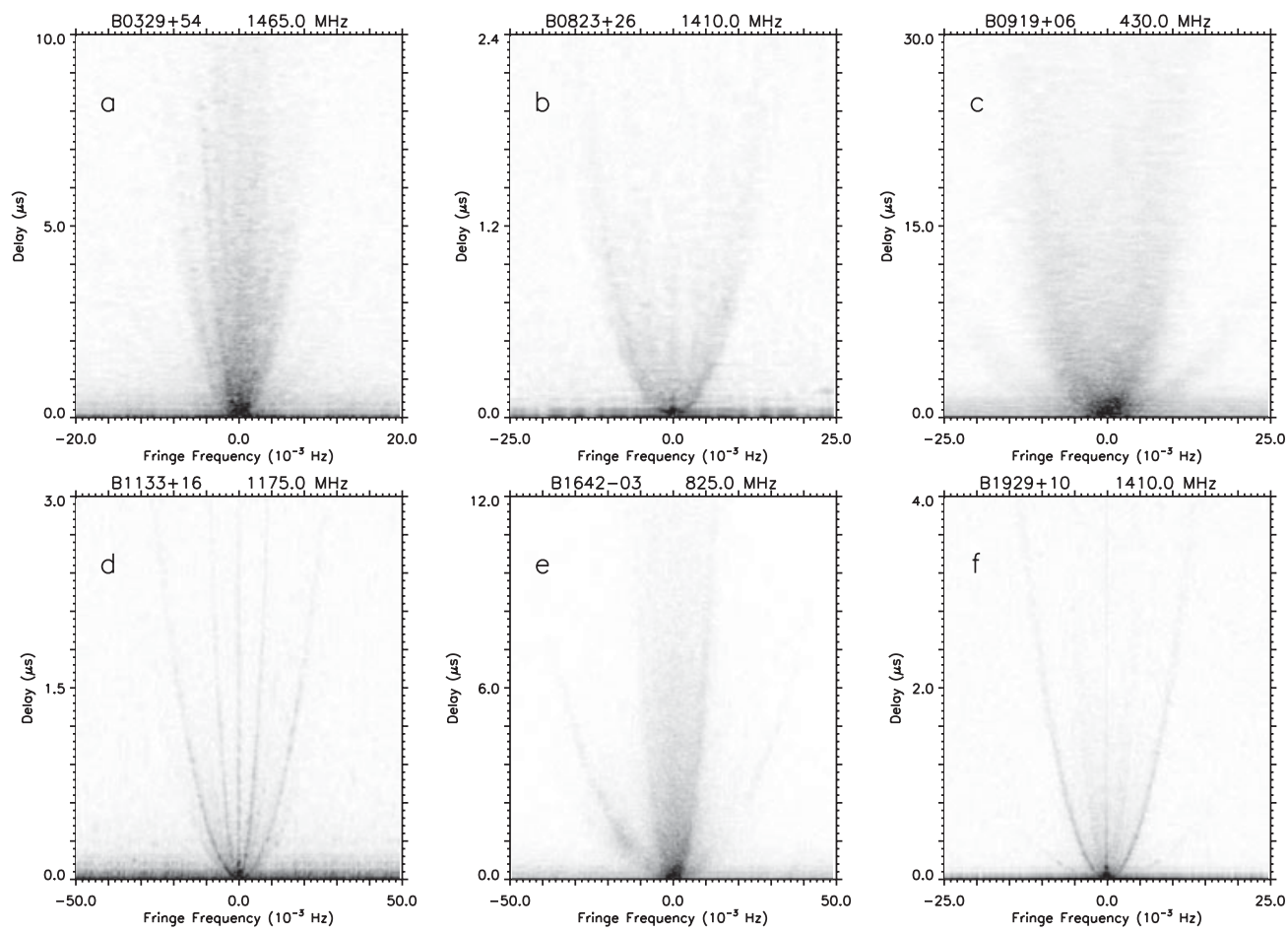

Figure 1. A sampling of six relatively nearby, strong pulsars, most of which show sharply delineated scintillation arcs. Credit: Putney \& Stinebring 2006.

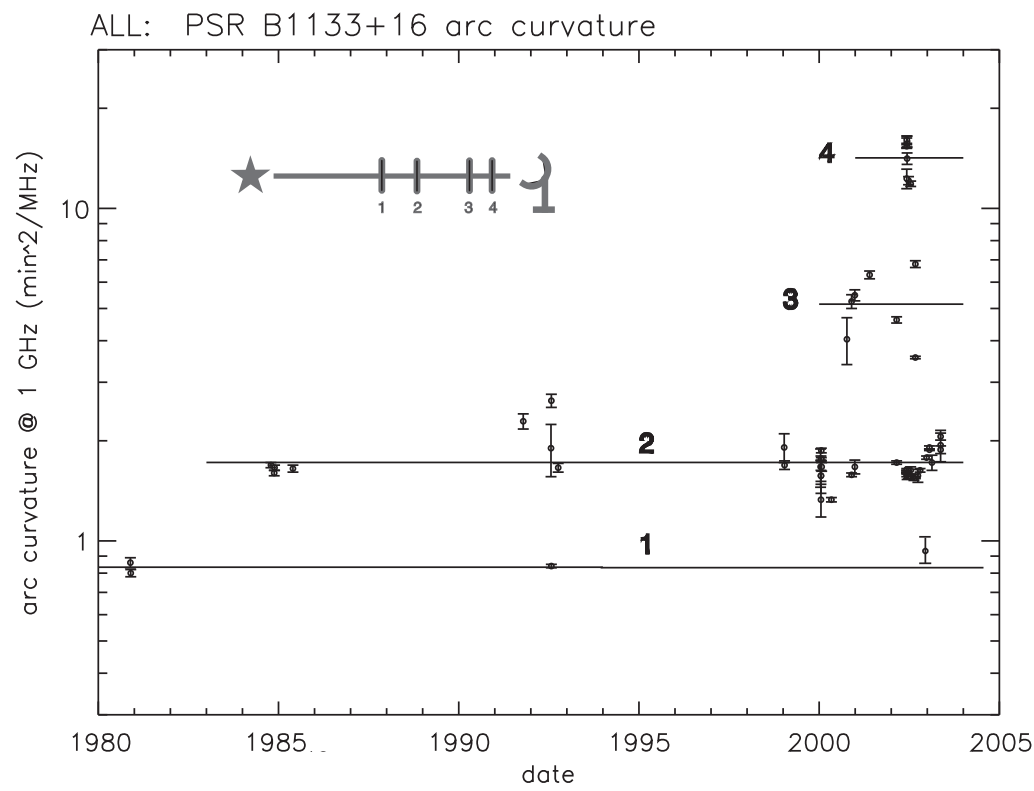

Figure 2. Evidence for four discrete scintillation arcs toward the relatively nearby $(d=350 \mathrm{pc})$ pulsar B1133+16. The scattering screen placements shown in the inset assume that the linear scattering structure is aligned with $V_{\text {eff }}$. Any misalignment will move the screen closer to the pulsar. Credit: Stinebring 2006. 


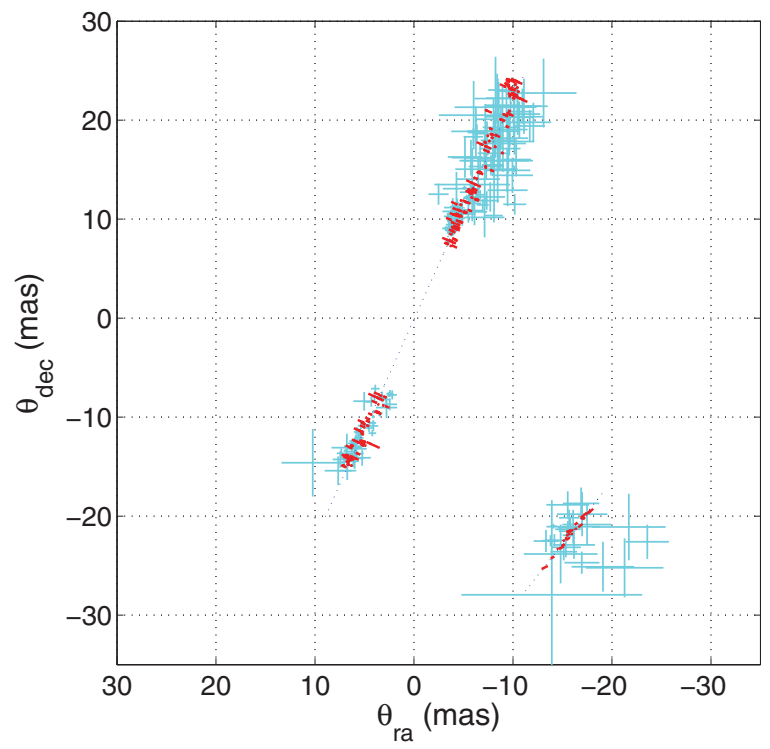

Figure 3. Scattering structures deduced by Brisken et al. 2010 (Figure 5) from VLBI observations of B0834+06 with Arecibo and the Green Bank Telescope.

In a following model (Liu et al. 2016) this was generalized to allow a double refraction event to explain the " $1 \mathrm{~ms}$ " feature in the Brisken et al. (2010) data (the region offset down and to the right in Fig. 3).

Despite the qualitatively compelling character of the Pen and Levin (2014) scenario, the model is lacking in physical detail. In particular, the tilt angle of the sheet is not well constrained, nor is the amplitude of the wavefield needed to scatter the rays to the observer.

\section{Toward a more quantitative model}

With Barney Rickett I have been working on a model that provides additional details and observational diagnostics in order to explain linear scattering features toward pulsars. A starting point for that model is to specify the relation between the deflection angle of a ray in the sheet and the width of the "glitter patch" that is produced on the surface (Fig. 4). Inspection of that figure shows that as the tilt angle $\delta$ of the wave is reduced the glitter patch becomes more nearly linear and narrower in width. The idea, as applied to the extremely small (milliarcsecond or less) transverse thickness of the scattering structures on the sky toward pulsars, is that small angle scattering off of these grazing incidence sheets can lead to a testable model. We have a multi-frequency, multi-epoch data Arecibo set for the pulsar B1133+16 against which we intend to test this model quantitatively.

\section{Conclusions}

We have shown evidence that scintillation arcs are more sharply delineated than would be expected for isotropic scattering. (This is but one line of evidence for anisotropic scattering in the ISM; see Rickett's contribution in this volume for further references.) A planar sheet geometry, intercepted at a grazing incidence angle, seems a generic producer 


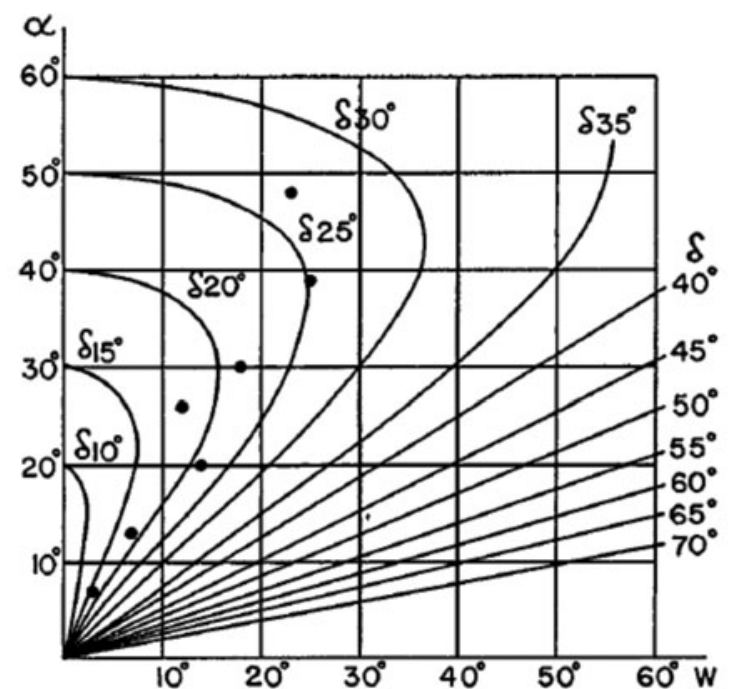

FIG. 6. Width of sun path; curves, theoretical, and dots, experimental.

Figure 4. Width $w$ of glitter patches on the surface of the ocean as a function of solar elevation $\alpha$ and parameterized by the maximum wave tilt $\delta$. Credit: Hulburt 1934 .

of such structures (Pen \& Levin 2014). Further details need to be added to that model to make it broadly predictive. We have sketched a way forward in this contribution.

\section{References}

Brisken, W. F., Macquart, J.-P., Gao, J. J., Rickett, B. J., Coles, W. A., Deller, A. T., Tingay, S. J., \& West, C. J. 2010, ApJ, 708, 232

Cordes, J. M., Rickett, B. J., Stinebring, D. R., \& Coles, W. A. 2006, ApJ, 637, 346

Hulburt, E. O. 1934, JOSA, 34, 24

Liu, S., Pen, U.-L., Macquart, J.-P., Brisken, W., \& Deller, A. 2016, MNRAS, 458, 1289

Pen, U.-L. \& Levin, Y. 2014, MNRAS, 442, 3338

Putney, M. L. \& Stinebring, D. R. 2006, Chi. JAA Sup., 6, 233

Romani, R. W., Blandford, R. D., \& Cordes, J. M. 1987, Nature, 328, 324

Stinebring, D. R. 2006, Chi. JAA Sup., 6, 200

Stinebring, D. R., McLaughlin, M. A., Cordes, J. M., Becker, K. M., Goodman, J. E. E., Kramer, M. A., Sheckard, J. L., \& Smith, C. T. 2001, ApJ, 549, L97

Walker, M. A., Melrose, D. B., Stinebring, D. R., \& Zhang, C. M. 2004, MNRAS, 354, 43 